\title{
Cirrhosis: Therapeutic Aspects and Outcome for Hospitalized Patients in Burkina Faso
}

\author{
Eric Nagaonlé Somé ${ }^{*}{ }^{\circledR}$, Félicité W. Nana1, Isabelle Télarpoa Lompo², Drabo Maxime1, \\ Roger Sombié2
}

${ }^{1}$ Institut de Recherche en Sciences de la Santé (IRSS), Biomedical and Public Health Department, Ouagadougou, Burkina Faso ${ }^{2}$ Université Joseph Ki-Zerbo, UFR des Sciences de la Santé, Gastro-Enterology Department, Ouagadougou, Bukina Faso Email: ^eric.some@gmail.com, felynanaw@gmail.com, telarpoa@yahoo.fr,mdrabok@gmail.com,docsomb@gmail.com

How to cite this paper: Somé, E.N., Nana, F.W., Lompo, I.T., Maxime, D. and Sombié, R. (2021) Cirrhosis: Therapeutic Aspects and Outcome for Hospitalized Patients in Burkina Faso. Open Journal of Epidemiology, 11, 152-162.

https://doi.org/10.4236/ojepi.2021.112014

Received: January 19, 2021

Accepted: May 8, 2021

Published: May 11, 2021

Copyright $\odot 2021$ by author(s) and Scientific Research Publishing Inc. This work is licensed under the Creative Commons Attribution International License (CC BY 4.0).

http://creativecommons.org/licenses/by/4.0/

\begin{abstract}
Introduction: Cirrhosis represents $27.63 \%$ of the hepatobiliary diseases in Burkina Faso. We aimed at studying the therapeutic and evolutionary features of the cirrhosis. Patients and methods: We implemented a cross-sectional and descriptive study, collecting retrospectively the data from 1st January 2012 to 31st March 2014. The diagnosis of cirrhosis was based on 1) clinical criteria (an edema-ascitic syndrome with a heterogeneous hepatomegaly with a sharp inferior border or atrophic liver and a portal hypertension); 2) biological criteria (a hepatocellular insufficiency syndrome); and 3) ultrasound imaging suggesting cirrhosis. Qualitative variables were presented as frequencies and percentages while quantitative ones were presented as means. Results: The data of 273 patients representing $33.9 \%$ of all hospitalizations were analyzed. The hepato-biliary diseases represented $74.7 \%$ of all diagnosis. The participants' mean age was 46.9 years and the sex ratio, 2.7. The HBs antigen and anti-HCV antibodies were positive in $76.5 \%$ and $14.6 \%$ of the cases, respectively. Ascites was treated with puncture in $40.2 \%$ of patients, a salt-free diet (38.8\%) and diuretics (54.2\%). Hepatic encephalopathy was treated with lactulose in $73.6 \%$ of patients and two patients (1.3\%) underwent esophageal varices ligation to treat their gastrointestinal bleeding. Lamivudine, tenofovir, and lamivudine-tenofovir combination were administered to $57.4 \%, 32.8 \%$, and $10 \%$ of HBs Antigen positive patients, respectively. Conclusion: Cirrhosis still mostly affects the young and active male population at the gastro-enterology department of the university teaching hospital Yalgado Ouédraogo. Patients show up at a very advanced stage of the disease in poorly prepared health centres. In this context, prevention by the anti-hepatitis $B$ immunization and early systematic screening with treatment when indicated are very efficient weapons at our disposal.
\end{abstract}




\section{Keywords}

Cirrhosis, Hepatitis B Virus, Treatment, Prognosis, Sub-Saharan Africa

\section{Introduction}

All chronic liver diseases can lead to cirrhosis, an irreversible lesion that combines fibrosis, a process of nodular regeneration and profound modification of the vascularization of the parenchyma. Among the many possible causes of cirrhosis, one of the most important is alcohol consumption, which is the main cause in developed countries [1] [2]. In contrast, in developing countries, chronic viral hepatitis is the leading cause [3] [4] [5] [6]. According to the WHO, 350 million people suffer from chronic liver disease in the world, and Africa has 60 million with a quarter of all deaths per year [7]. Indeed, Africa is considered with the Southeast Asia as a highly endemic area where the prevalence of chronic hepatitis B is estimated at least at $8 \%$. In Burkina Faso, the prevalence of chronic carriage of the hepatitis B surface antigen (HbsAg) is estimated at $9.1 \%$ [8], despite the existence of an effective vaccine which should have contributed to reducing the incidence of the hepatitis B. In 2002, in Burkina Faso, cirrhosis dominated hepatobiliary pathologies with $27.63 \%$ of cases. In addition, these conditions affect young and active subjects, which hinders the emergence of already fragile economies [9]. There are several curative treatments that allow stabilization or even a regression of the lesions. The cases management is limited to the prevention of the occurrence of complications. But the effective curative treatment remains the liver transplant. We aimed to study the therapeutic and evolutionary aspects of cirrhosis at the university teaching hospital Yalgado Ouedraogo (UTH-YO) in Ouagadougou, the capital city of Burkina Faso.

\section{Patients and Methods}

A cross-sectional descriptive study with a retrospective data collection, from January 1st, 2012 to March 31st, 2014 was implemented in the hepato-gastroenterology department of the UTH-YO in Ouagadougou. All records of patients diagnosed with cirrhosis during the study period were included. The cirrhosis was diagnosed on the basis of 1) clinical criteria (edemato-ascitic syndrome, heterogeneous hepatomegaly with a sharp lower edge or atrophic liver, portal hypertension); 2) biological criteria (hepatocellular insufficiency syndrome); and 3) medical imaging (ultrasound and CT scan) suggesting cirrhosis. Patients whose records were incomplete or could not be used were excluded. A data collection form was used as the data collection tool. The form included an identification section, a clinical, and biological, variables section as well as the imaging results parts and other investigations' results such as the endoscopy and the CT scan data. Finally the form included also data on the treatments and the pro- 
gression of the disease under treatment. A descriptive analysis was implemented and qualitative variables were pooled as frequencies and percentages while the quantitative ones were calculated as means with standard deviations. Data were entered and analyzed using EPI-INFO software, version 3.5.3 and EXCEL 2010.

\section{Results}

\subsection{Sociodemographic Characteristics}

Overall, 803 patients were hospitalized in the hepato-gastroenterology department during the study period. Hepatobiliary pathology represented $74.7 \%$ of the cases. The data of 273 patients (33.9\% of hospitalizations) were included in the analysis.

The mean age ( \pm standard deviation) was $46.9( \pm 13.8)$ with a range of $16-82$ years. The most represented age group was $41-60$ years. The sex ratio was 2.7 . Farmers and housewives accounted for $34.2 \%$ (93/272) of the patients (Table 1 ). The mean duration from the first clinical manifestations to medical visit was 99 days.

\subsection{Etiologies and Seriousness of Cirrhosis}

Overall, 239 (87.5\%) patients, were screened for HBsAg and 183 (76.5\%) tested positive while 191 (69.9\%) were screened for anti-hepatitis $\mathrm{C}$ virus antibodies (anti-HCVAb) and 28 (14.6\%) were tested positive. Viral load was performed for $48(17.5 \%)$ HBsAg+ patients and the mean viral load was 23,295,569.7 IU/ml, with a range of 23 to $110,000,000 \mathrm{IU} / \mathrm{mL}$ and was undetectable in two patients. C-RNA (HCV) was performed in $04(14.2 \%)$ of the 28 anti-HCV Ab+ patients with a mean viral load of $2749 \mathrm{IU} / \mathrm{ml}$.

Table 1. Socio-demographic characteristics.

\begin{tabular}{ccc}
\hline variable & Frequency & Percent (\%) \\
\hline Sex $=$ female & 73 & 26.74 \\
Age group & & \\
$0-20$ & 4 & 1.46 \\
$21-40$ & 101 & 37.00 \\
$41-60$ & 116 & 42.5 \\
$>60$ & 52 & 19.04 \\
Occupation & & \\
Pupil/Student & 13 & 4.76 \\
Famers & 49 & 17.95 \\
Civil servants & 44 & 16.12 \\
Housewives & 44 & 16.12 \\
Retired & 5 & 1.83 \\
Informal sector & 33 & 12.09 \\
Not specified & 85 & 31.13 \\
\hline
\end{tabular}


The results of various non-specific laboratory tests, the dosage of prothrombin, concentration and serum albumin (protidogramme) and previous clinical examination were used to classify 28 patients according to Child Pugh categories [10] (https://en.wikipedia.org/wiki/Child\%E2\%80\%93Pugh score). Fourteen, 11 and three patients were in Child Pugh's classes C, B or A, respectively.

\subsection{The Therapeutic Features}

\section{Symptomatic treatments}

To treat ascites, punctures were realized in $110(40.2 \%)$ patients with a mean fluid quantity of $3.5 \mathrm{~L} \pm 1.3$ per patient. A salt-free diet and diuretic medications were administered to $38.8 \%$ and $54.2 \%$ of the cases, respectively.

Lactulose was used in $73.6 \%$ of cases as a treatment for both inaugural hepatic encephalopathy and encephalopathy occuring during hospitalization. Twenty-four (16.2\%) patients received emergency transfusion during acute episodes of anaemia at admission or during hospitalization. The gastrointestinal bleeding by rupture of esophageal varices was treated by ligation in $02(1.3 \%)$ patients.

Etiological treatment and efficacy of the treatment

The main cause of cirrhosis was $\mathrm{HBV}$ ( $\mathrm{HBsAg}+$ in $76.5 \%$ of cases). In our study, the main used antivirals were lamivudine which was administered to 35 patients $(57.4 \%)$ and tenofovir to $20(32.8 \%)$ patients. Tenofovir has been combined with lamivudine in six $(9.8 \%)$ patients.

Using lamivudine, the virological response was complete (DNA-HBV undetectable) in 08 patients $(72.7 \%)$ among those whose viral load was performed regularly. Four patients (6.5\%) developed resistance. With tenofovir, the virological response was complete in 07 patients (87.5\%). With the lamivudine-tenofovir combination, the virological response was complete in all patients.

With regard to the biochemical response, the ALAT returned to normal range in $63.6 \%, 75 \%$ and $100 \%$ of lamivudine, tenofovir, and tenofovir-lamivudine treated patients, respectively. The duration of the hospitalization ranged from 0 to 35 days with a mean duration of $8.5 \pm 6.3$ days. The different treatments administered and their outcomes, the duration of spontaneous survival of those who died are shown in Table 2.

\section{Discussion}

\subsection{The Study Limitations}

Our study has some limitations which are specific to retrospective studies in general including the missing data. An important missing data was the direct cause of death. This gap did not allow us to identify the most frequent causes of cirrhosis mortality in our context. However, the robustness of the variables collected as well as the descriptive nature of our study minimized the impact of this gap. 
Table 2. The different treatments received by patients.

\begin{tabular}{ccc}
\hline Treatment's option & Frequency & Percent (\%) \\
\hline Salt-free diet & 106 & 38.8 \\
Ascites puncture & 110 & 40.2 \\
Pain-killers & 148 & 54.2 \\
Lactulose (duphalac) & 160 & 58.6 \\
Blood transfusion & 201 & 73.6 \\
Treatment's outcome & 24 & 08.7 \\
Improved status (patients discharged) & 128 & \\
Discharge against medical advice & 60 & 46.88 \\
Death & 85 & 22.00 \\
Spontaneous survival (n $=68)$ & & 31.13 \\
$<1$ month & 31 & \\
1 - 3 months & 22 & 45.58 \\
$3-6$ months & 09 & 32.35 \\
6 - 9 months & 01 & 13.35 \\
9 - 12 months & 02 & 1.47 \\
$>12$ months & & 2.94 \\
\hline
\end{tabular}

\subsection{Sociodemographic, Epidemiological and Etiological Aspects}

The mean age of our patients was $46.9 \pm 13.8$ years. It was similar to the mean age reported by other published studies [11] or not published ones [12] [13] in Burkina Faso. These results showed that African patients are younger, as compared to patients in developed countries where the mean age ranges between 51 58 years [13] [14]. The difference in risk factors for cirrhosis in developed countries (predominance of alcohol consumption and delayed onset of the disease) compared to limited resources settings (predominance of viral hepatitis infection which is acquired early in the first years of life) may explain this difference in the mean ages [2] [4] [6].

As to the gender, it is established that the liver diseases (infections or cancers) are predominantly male's pathologies [15] [16] [17] [18]. The sex ratio of 2.7 found in our work once again corroborated this observation. The male gender would be associated with a greater mortality from bleeding caused by ruptured esophageal varices [19]. Regarding the causes of cirrhosis, the in-hospital proportions of patients testing positive for HBsAg and anti-VHCAb was $76.5 \%$ and $14.6 \%$, respectively, as in many other studies [11] [13].

The mean duration between the onset of the first symptoms and the first medical visit was 99 days. This long delay could in itself explain the advanced stage of the disease when the patients show up: $50 \%$ of them were already in the Child Pugh's class C whereas the mean viral load was 23,295,569, $7 \mathrm{IU} / \mathrm{ml}$ [20] [21]. 


\subsection{Therapeutics Aspects}

\section{Symptomatic treatment}

Ascites is one of the most common complications that often reveals cirrhosis [22] [23]. In our study, $40.2 \%$ of patients with ascites received a puncture, $38.8 \%$ observed a salt-free diet and $54.2 \%$ took diuretics. In the literature, it has been shown that a low-sodium diet combined with diuretics intake was very effective in $90 \%$ of cases in the treatment of ascites [23]. When ascites is refractory (ascites not responding to treatment with diuretics associated with the salt-free diet or relapsing after high doses of diuretic [23] [24], other therapies can be initiated including repeated ascites punctures, peritoneo-venous shunts or abdominal drainage. Shunts and drainage have the advantage to better control ascites, reducing frequent hospitalizations and the financial burden on patients and the healthcare system, and improving patients' lives without anyway significantly affecting their survival as compared to traditional punctures [22] [24]. These interventions should be assessed in a broader context of palliative care for end-of-life patients with chronic liver disease [22].

Hepatic encephalopathy with an unfavorable outcome as per the West Haven criteria [25] is associated with higher short-term mortality (within 90 days) [26]. The treatment of encephalopathy is primarily based on the treatment (if possible) of the triggering cause. In addition to nutrition, the use of disaccharides and non-absorbable antibiotics (rifaximin) are part of the therapeutic means [25]. Treatments aiming to modify the colonic bacterial flora and accelerate intestinal transit may prove efficacious. The most commonly used treatment is the lactulose or lactilol (non-absorbable disaccharide) [25] [26] [27]. In our study, this treatment was administered in $73.6 \%$ of the cases. However, treatment combining lactulose and rifaximin would be more efficacious (incidence rate ratio (IRR) $=0.28$ confidence interval $(\mathrm{CI}) 95 \%(0.27-0.30))$ than lactulose alone (IRR = $0.3195 \%$ CI $(0.30-0.32))$, itself more effective than rifaximin alone (IRR + 0.49 95\% CI (0.45 - 0.53)) [28].

Another serious complication of cirrhosis is the gastrointestinal bleeding from ruptured oesophageal varices. Its incidence is estimated at between $12 \%$ and $15 \%$ per year with a mortality rate at up to $20 \%$ [19] [29]. Different therapeutic procedures are used including drug treatments (non-cardio selective beta-blockers, or isosorbide mononitrate or both), endoscopic interventions (ligation of oesophageal varices, or sclerotherapy), blood shunts (total shunt, transjugular portosystemic or distal splenorenal shunt). The superiority of an intervention over another has not been demonstrated [30]. However, the best treatment remains the prevention by treating the cause of the cirrhosis (alcohol withdrawal or effective antiviral treatment) [31]. In our study the ligation of esophageal varices was performed in 2 patients $(8.3 \%)$. This low frequency of ligation could be explained by the high cost of the needed material to implement the intervention.

Etiological treatment

Antiviral therapy with nucleoside analogue has been systematically prescribed 
every time it was indicated as suggested in the literature [7]. However, only $23.3 \%$ of all eligible patients were treated mainly because of financial constraints. A previous study in the same setting a few years ago found similar results with $20.3 \%$ of the patients in need of antiviral treatment, able to afford the cost of the medicines [7]. Actually in Burkina Faso, the hepatitis B infection does not benefit from health policy easing the access to the antivirals as it is provided for HIV patients.

Antiviral molecules used to treat hepatitis B include tenofovir, entecavir, and lamivudine [32] [33] [34]. A total of $57.4 \%$ of our patients were treated with lamivudine. Sombié [7] found a higher proportion (96.7\%) of patients on lamivudine as antiviral to treat the HBV. At the time of his study, tenofovir was not yet introduced in Burkina Faso.

The efficacy of lamivudine to slow down the HBV-born cirrhosis has been demonstrated. However, it is no more currently recommended by the WHO because of its weak virological barrier and the quick emergence of resistant strains [33]. Indeed, the main drawback of lamivudine is its high risk of resistance $(20 \%$ per year) [7]. In our study, we noted resistance to lamivudine in $6.5 \%$ of the cases. This low rate of resistance was also noted by Sombié [7] (6.7\% of his cases). This would be explained by the small number of treated patients and the shorter duration of the follow-up.

Overall, $32.8 \%$ of patients received tenofovir. With tenofovir, HBV-DNA was undetectable in $87.5 \%$ of the cases. This proportion was within the range of the complete virological response reported and which is greater than $76 \%$ and was higher than the result found by Yao [35] who reported $72.7 \%$. No cases of resistance to tenofovir were observed in our study. This result confirms the data in the literature which have not reported any case of resistance to tenofovir to date [36].

Tenofovir was administered in combination with lamivudine to $9.8 \%$ of the patients. This proportion was higher than the $6.4 \%$ observed by Sombié [7]. The viral load was undetectable in all six patients treated with a lamivudine-tenofovir combination (including four patients who faced resistance to lamivudine).

With respect to the biochemical response, the ALAT returned to normal range in $63.6 \%$ of the lamivudine-treated patients. This proportion was comparable to SIA's result from a study implemented in the same hospital [37] in Burkina, and comparable to Yao's [35] in China, which reported biochemical response rates of $71.8 \%$ and $79.1 \%$, respectively. Under tenofovir this rate was $75 \%$, similar to the rates of $77 \%$ within one year of treatment in the literature [35].

\subsection{The Disease Progression}

In our study, the duration of follow-up was relatively short, with a mean duration of $8.5 \pm 6.3$ days. Actually, once the diagnosis is known, and after they experience some relief of their symptoms, most patients were discharged because they could no longer afford to pay for the care and the hospitalization. Most of them requested to be discharged. Before our study, Tinto and Sawadogo respec- 
tively, found the same result with $90.4 \%$ and $100 \%$ of the hospitalized patients staying less than one month [12] [13]. The in-hospital case fatality rate was high at $32.3 \%$ comparable to the results of previous studies in the same setting which were between $28.5 \%$ and $33.3 \%$ [12] [13]. The causes of this high mortality would mainly be the gastrointestinal bleeding and hepatic encephalopathy [19] [26] [28].

\section{Conclusion}

In the gastroenterology department of the UTH-YO, cirrhosis was a very frequent reason for medical visit and hospitalization. It affects the male, young and active population. Patients show up very late at an advanced stage of the disease. The hepatitis $\mathrm{B}$ and $\mathrm{C}$ viruses are the most frequent etiologies. The available therapeutic means are quite limited, due to an under-equipped health system, but also to very poor patients. This context leads to an excess lethality by cirrhosis and a shorter survival time. A survival analysis on a cohort of cirrhotic patients during a longer period of follow up in our LMIC context may better highlight the details of the disease progression as well as the risk factors for death. The prevention by vaccination (anti-hepatitis B) and early systematic screening with treatment when indicated are very efficient and effective weapons at our disposal.

\section{Conflicts of Interest}

The authors declare no conflicts of interest regarding the publication of this paper.

\section{References}

[1] Askgaard, G., Gronbaek, M., Kjaer, M.S., Tjonneland, A. and Tolstrup, J.S. (2015) Alcohol Drinking Pattern and Risk of Alcoholic Liver Cirrhosis: A Prospective Cohort Study. Journal of Hepatology, 62, 1061-1067. https://doi.org/10.1016/j.jhep.2014.12.005

[2] Stokkeland, K., Hilm, G., Spak, F., Franck, J. and Hultcrantz, R. (2008) Different Drinking Patterns for Women and Men with Alcohol Dependence with and without Alcoholic Cirrhosis. Alcohol and Alcoholism, 43, 39-45. https://doi.org/10.1093/alcalc/agm135

[3] Mendy, M.E., Welzel, T., Lesi, O.A., Hainaut, P., Hall, A.J., Kuniholm, M.H., et al. (2010) Hepatitis B Viral Load and Risk for Liver Cirrhosis and Hepatocellular Carcinoma in the Gambia, West Africa. Journal of Viral Hepatitis, 17, 115-122. https://doi.org/10.1111/j.1365-2893.2009.01168.x

[4] Kuniholm, M.H., Lesi, O.A., Mendy, M., Akano, A.O., Sam, O., Hall, A.J., et al. (2008) Aflatoxin Exposure and Viral Hepatitis in the Etiology of Liver Cirrhosis in the Gambia, West Africa. Environmental Health Perspectives, 116, 1553-1557. https://doi.org/10.1289/ehp.11661

[5] Surial, B., Wyser, D., Béguelin, C., Ramírez-Mena, A., Rauch, A. and Wandeler, G. (2021) Prevalence of Cirrhosis in Individuals with Hepatitis B Virus Infection in Sub-Saharan Africa: Systematic Review and Meta-Analysis. Liver International, 41, 710-719. https://doi.org/10.1111/liv.14744 
[6] Vento, S., Dzudzor, B., Cainelli, F. and Tachi, K. (2018) Liver Cirrhosis in Sub-Saharan Africa: Neglected, Yet Important. The Lancet Global Health, 6, E1060-E1061. https://doi.org/10.1016/S2214-109X(18)30344-9

[7] Sombié, R., Bougouma, A., Diallo, O., Bonkoungou, G., Cissé, R., Sangare, L., et al. (2010) Hépatite B chronique: Aspects épidémiologique, diagnostique, thérapeutique et évolutif au centre hospitalier universitaire Yalgado Ouédraogo de Ouagadougou. Journal Africain d' Hépato-Gastroentérologie, 4, 3-10. https://doi.org/10.1007/s12157-009-0137-2

[8] Meda, N., Tuaillon, E., Kania, D., Tiendrebeogo, A., Pisoni, A., Zida, S., et al. (2018) Hepatitis B and C Virus Seroprevalence, Burkina Faso: A Cross-Sectional Study. Bulletin of the World Health Organization, 96, 750-759. https://doi.org/10.2471/BLT.18.208603

[9] Flemming, J.A., Dewit, Y., Mah, J.M., Saperia, J., Groome, P.A. and Booth, C.M. (2019) Incidence of Cirrhosis in Young Birth Cohorts in Canada from 1997 to 2016: A Retrospective Population-Based Study. The Lancet Gastroenterology \& Hepatology, 4, 217-226. https://doi.org/10.1016/S2468-1253(18)30339-X

[10] D’Arienzo, A., Manguso, F., Scaglione, G., Vicinanza, G., Bennato, R. and Mazzacca, G. (1998) Prognostic Value of Progressive Decrease in Serum Cholesterol in Predicting Survival in Child-Pugh C Viral Cirrhosis. Scandinavian Journal of Gastroenterology, 33, 1213-1218. https://doi.org/10.1080/00365529850172593

[11] Nacoulma, E.W.C., Zongo, S., Drabo, Y.J. and Bougouma, A. (2007) Les différents types d'anémies au cours des cirrhoses au centre hospitalier universitaire Yalgado Ouédraogo de Ouagadougou (Burkina Faso). Cahiers Santé, 17, 87-91.

[12] Sawagodo, S.I.A. (2011) Cirrhose décompensée et carcinome hépatocellulaire au centre hospitalier universitaire Yalgado Ouédraogo de Ouagadougou: Aspects épidémiologiques, cliniques et thérapeutiques. Thèse de Médecine, Université de Ouagadougou, Ouagadougou, 1-120.

[13] Tinto, S. (2002) Cirrhoses et cancers primitives du foie au centre hospitalier national Yalgado Ouédraogo de Ouagadougou: Aspects épidémiologiques, cliniques et évolutifs. Thèse en médecine, Ouagadougou. Thèse de Médecine, Université de Ouagadougou, Ouagadougou, 30-45.

[14] Beste, L.A., Leipertz, S.L., Green, P.K., Dominitz, J.A., Ross, D. and Ioannou, G.N. (2015) Trends in Burden of Cirrhosis and Hepatocellular Carcinoma by Underlying Liver Disease in US Veterans, 2001-2013. Gastroenterology, 149, 1471-1482.E5. https://doi.org/10.1053/j.gastro.2015.07.056

[15] Shimizu, I., Kamochi, M., Yoshikawa, H. and Nakayama, Y. (2012) Gender Difference in Alcoholic Liver Disease. In: Shimizu, I., Ed., Trends in Alcoholic Liver Disease Research-Clinical and Scientific Aspects, IntechOpen, London.

https://www.intechopen.com/books/trends-in-alcoholic-liver-disease-research-clini cal-and-scientific-aspects/gender-difference-in-alcoholic-liver-disease https://doi.org/10.5772/27178

[16] Guy, J. and Peters, M. (2013) Liver Disease in Women: The Influence of Gender on Epidemiology, Natural History, and Patient Outcomes. Gastroenterology \& Hepatology, 9, 633-639.

[17] Sagnelli, E., Stroffolini, T., Sagnelli, C., Pirisi, M., Babudieri, S., Colloredo, G., et al. (2018) Gender Differences in Chronic Liver Diseases in Two Cohorts of 2001 and 2014 in Italy. Infection, 46, 93-101. https://doi.org/10.1007/s15010-017-1101-5

[18] Greten, T.F. (2019) Gender Disparity in HCC: Is It the Fat and Not the Sex? Journal of Experimental Medicine, 216, 1014-1015. https://doi.org/10.1084/jem.20190441 
[19] Haukeland, J.W., Smastuen, M.C., Palsdatter, P.P., Ismail, M., Konopski, Z., Jorgensen, K.K., et al. (2020) Effect of Gender on Mortality and Causes of Death in Cirrhotic Patients with Gastroesophageal Varices. A Retrospective Study in Norway. PLoS ONE, 15, e0230263. https://doi.org/10.1371/journal.pone.0230263

[20] Patel, N., Yopp, A. and Singal, A. (2015) Diagnostic Delays Are Common Among Patients with Hepatocellular Carcinoma. Journal of the National Comprehensive Cancer Network, 13, 543-549. https://doi.org/10.6004/jnccn.2015.0074

[21] Fu, B., Wang, W. and Shi, X. (2015) Impact of Delayed Diagnosis Time in Estimating Progression Rates to Hepatitis C Virus-Related Cirrhosis and Death. Statistical Methods in Medical Research, 24, 693-710. https://doi.org/10.1177/0962280211424667

[22] Macken, L., Joshi, D., Messenger, J., Austin, M., Tibble, J., Mason, L., et al. (2017) Palliative Long-Term Abdominal Drains in Refractory Ascites Due to End-Stage Liver Disease: A Case Series. Palliative Medicine, 31, 671-675. https://doi.org/10.1177/0269216316671281

[23] Singhal, S., Baikati, K., Jabbour, I. and Anand, S. (2012) Management of Refractory Ascites. American Journal of Therapeutics, 19, 121-132. https://doi.org/10.1097/MJT.0b013e3181ff7a8b

[24] Macken, L., Bremner, S., Gage, H., Touray, M., Williams, P., Crook, D., et al. (2020) Randomised Clinical Trial: Palliative Long-Term Abdominal Drains vs Large-Volume Paracentesis in Refractory Ascites Due to Cirrhosis. Alimentary Pharmacology \& Therapeutics, 52, 107-122. https://doi.org/10.1111/apt.15802

[25] Fallahzadeh, M.A. and Rahimi, R.S. (2020) Hepatic Encephalopathy and Nutrition Influences: A Narrative Review. Nutrition in Clinical Practice, 35, 36-48. https://doi.org/10.1002/ncp.10458

[26] Maggi, D., Borgonovo, A., Bansho, E., Soares-Silva, P., Silva, T., Colombo, B., et al. (2019) Serial Assessment of Hepatic Encephalopathy in Patients Hospitalised for Acute Decompensation of Cirrhosis. Annals of Hepatology, 18, 331-337. https://doi.org/10.1016/j.aohep.2018.11.002

[27] Patidar, K.R. and Bajaj, J.S. (2015) Covert and Overt Hepatic Encephalopathy: Diagnosis and Management. Clinical Gastroenterology and Hepatology, 13, 2048-2061. https://doi.org/10.1016/j.cgh.2015.06.039

[28] Tapper, E.B., Aberasturi, D., Zhao, Z., Hsu, C.-Y. and Parikh, N.D. (2020) Outcomes after Hepatic Encephalopathy in Population-Based Cohorts of Patients with Cirrhosis. Alimentary Pharmacology \& Therapeutics, 51, 1397-1405. https://doi.org/10.1111/apt.15749

[29] Yoo, J.J., Kim, S.G., Kim, Y.S., Lee, B., Jeong, S.W., Jang, J.Y., et al. (2020) Propranolol plus Endoscopic Ligation for Variceal Bleeding in Patients with Significant Ascites: Propensity Score Matching Analysis. Medicine, 99, e18913. https://doi.org/10.1097/MD.0000000000018913

[30] Simonetti, R.G., Perricone, G., Robbins, H.L., Battula, N.R., Weickert, M.O., Sutton, R., et al. (2020) Portosystemic Shunts versus Endoscopic Intervention with or without Medical Treatment for Prevention of Rebleeding in People with Cirrhosis. The Cochrane Database of Systematic Reviews, No. 10, CD000553. https://doi.org/10.1002/14651858.CD000553.pub3

[31] Moon, A.M., Green, P.K., Rockey, D.C., Berry, K. and Ioannou, G.N. (2020) Hepatitis C Eradication with Direct-Acting Anti-Virals Reduces the Risk of Variceal Bleeding. Alimentary Pharmacology \& Therapeutics, 51, 364-373. https://doi.org/10.1111/apt.15586 
[32] Terrault NA, Lok ASF, McMahon BJ, Chang KM, Hwang JP, Jonas MM, et al. (2018) Update on Prevention, Diagnosis, and Treatment of Chronic Hepatitis B: AASLD 2018 Hepatitis B Guidance. Hepatology, 67, 1560-1599. https://doi.org/10.1002/hep.29800

[33] World Health Organization (2015) Guidelines for the Prevention, Care and Treatment of Persons with Chronic Hepatitis B Infections. World Health Organization, 1-166.

[34] de Fraga, R.S., Van Vaisberg, V., Mendes, L.C.A., Carrilho, F.J. and Ono, S.K. (2020) Adverse Events of Nucleos(t)ide Analogues for Chronic Hepatitis B: A Systematic Review. Journal of Gastroenterology, 55, 496-514. https://doi.org/10.1007/s00535-020-01680-0

[35] Yao, G.B., Zhu, M., Cui, Z.Y., Wang, B.E., Yao, J.L. and Zeng, M.D. (2009) A 7-Year Study of Lamivudine Therapy for Hepatitis B Virus e Antigen-Positive Chronic Hepatitis B Patients in China. Journal of Digestive Diseases, 10, 131-137. https://doi.org/10.1111/j.1751-2980.2009.00375.x

[36] van Bommel, F., Zollner, B., Sarrazin, C., Spengler, U., Huppe, D., Moller, B., et al. (2006) Tenofovir for Patients with Lamivudine-Resistant Hepatitis B Virus (HBV) Infection and High HBV DNA Level during Adefovir Therapy. Hepatology, 44, 318-325. https://doi.org/10.1002/hep.21253

[37] Sia, R. (2009) Les hépatites virales chroniques au Centre hospitalier Universitaire Yalgado Ouédraogo: Aspects épidémiologiques, diagnostiques, thérapeutiques et évolutifs. Thèse de Ouagadougou (PA), Université de Ouagadougou, Ouagadougou, $1-77$. 